\title{
Análise do acúmulo de sedimentos por batimetria em lagoa facultativa no município de Campo Mourão/PR
}

As lagoas facultativas são utilizadas como pós-tratamento de efluentes ainda não estabilizados, e têm como mecanismo de ação uma associação entre diferentes microrganismos que se encontram em diferentes estrados do perfil. Quando não operadas de maneira eficiente, podem apresentar problemas como zonas mortas e zonas de curto-circuito. Desta forma, o objetivo deste estudo é analisar o acúmulo de sedimentos por meio de batimetria em uma lagoa facultativa no município de Campo Mourão. A coleta dos dados batimétricos foi realizada em um bote de alumínio, com o auxílio de uma baliza graduada acoplada a um disco de alumínio, e os pontos de coleta se distanciaram $5 \times 5 \mathrm{~m}$ e 10×5 metros (linha X ponto). A partir dos dados obtidos foram elaborados diagramas mostrando as cotas de altura do lodo no leito e a representação tridimensional do acúmulo de sedimentos. Notou-se com estas representações que a região mais atingida pelo fenômeno é o início da lagoa, onde se encontra a margem curvada, a qual influencia diretamente no fluxo do efluente. Por meio desta análise, concluiu-se que a sedimentação irregular na lagoa facultativa está associada ao seu formato, diferente do projetado, e à velocidade de entrada do fluido na lagoa, que é insuficiente para conduzir as partículas em suspensão por todo o leito da lagoa.

\section{Analysis of sediment accumulation by bathymetry in an optional lagoon in the municipality of Campo Mourão/PR}

\begin{abstract}
Facultative lagoons are used as post-treatment of unstabilized effluents, and they have as mechanism of action an association among different microorganisms located in different profile stages. When not operated efficiently, they can present problems such as dead zones and short circuit zones. Therefore, the objective of this study is to analyze sediment accumulation by bathymetry in a facultative lagoon in the city of Campo Mourão, Brazil. Bathymetric data were collected in an aluminum dinghy, with the aid of a ranging rod attached to an aluminum disc, and the collection points were $5 \times 5$ and $10 \times 5$ meters (line $X$ point). From the obtained data, diagrams were prepared showing the heights of the sludge in the bed and the three-dimensional representation of sediment accumulation .With these representations, it was noted that the most affected region is the beginning of the lagoon, where the curved margin is located, which directly influences the effluent flow. From this analysis, it was concluded that irregular sedimentation in the facultative lagoon is associated with both its shape that is different from the projected shape, and the entry fluid velocity into the lagoon, which is insufficient to carry the suspended particles throughout the lagoon bed.
\end{abstract}

Keywords: Bathymetry; Facultative pond; Wastewater treatment.

Topic: Engenharia Ambiental

Reviewed anonymously in the process of blind peer.
Received: 21/12/2019

Approved: 11/01/2020
Leandro Conti Garcia (iD

Universidade Tecnológica Federal do Paraná, Brasil http://lattes.cnpq.br/3627938742144763 http://orcid.org/0000-0002-1602-4336

lecgarciaa@gmail.com

Flávia Aparecida Reitz Cardoso (iD

Universidade Tecnológica Federal do Paraná, Brasil

http://lattes.cnpq.br/2663975071704461

http://orcid.org/0000-0002-0432-9191

flaviareitz@gmail.com

Fábio Rodrigo Kruger (iD)

Universidade Tecnológica Federal do Paraná, Brasil

http://lattes.cnpq.br/6622145568497966

http://orcid.org/0000-0002-6364-5741

fabiokruger@utfpr.edu.br
Vitoria Sapia Guerra (iD)

Universidade Tecnológica Federal do Paraná, Brasil

http://lattes.cnpq.br/5864954578814482

http://orcid.org/0000-0002-6473-4914

vitoriaguerra@alunos.utfpr.edu.br

Eudes José Arantes (ii)

Universidade Tecnológica Federal do Paraná, Brasil

http://lattes.cnpq.br/5368039952110556

http://orcid.org/0000-0002-7684-3069

eudesarantes@utfpr.edu.br
Referencing this:

GARCIA, L. C.; CARDOSO, F. A. R.; KRUGER, F. R.; GUERRA, V. S.; ARANTES, E. J.. Análise do acúmulo de sedimentos por batimetria em lagoa facultativa no município de Campo Mourão/PR. Revista Ibero Americana de Ciências Ambientais, v.11, n.1, p.177-183, 2020. DOI: http://doi.org/10.6008/CBPC2179-6858.2020.001.0017 


\section{INTRODUÇÃO}

As lagoas facultativas são utilizadas como pós-tratamento de efluentes que necessitam atingir os parâmetros legais para o lançamento em corpos hídricos. Estão inseridas em um cenário que abrange a biodigestão aeróbia e anaeróbia e suas características físicas, na implantação, envolvem uma construção com grande área superficial e profundidade em torno de dois metros. Estes atributos possibilitam grande influência da irradiação solar na superfície, além de permitir o desenvolvimento de uma camada de lodo no fundo, criando um ambiente anaeróbio (LIMA et al., 2018).

O desenvolvimento do consumo de matéria orgânica nas lagoas facultativas está ligado à associação de diversos organismos com nichos ecológicos diferentes. Devido à grande área de contato com a irradiação solar, algas se desenvolvem na metade superior do perfil da lagoa, e a produção de oxigênio por elas permite o crescimento de bactérias aeróbias. Estas bactérias se alocam próximo à superfície da lagoa e são responsáveis por degradar a DBO em suspensão (VON SPERLING, 2017).

A matéria orgânica não solúvel se deposita no fundo da lagoa, local não alcançado pela luz solar. Como esta situação impede o desenvolvimento das algas, acaba por inibir a oxigenação no manto de lodo e possibilita o desenvolvimento das bactérias anaeróbias que degradarão o restante da DBO presente no efluente. Este sistema dá ao efluente tratado estabilidade, não sendo necessários assim tratamentos posteriores (METCALF et al., 2015). A deposição de sólidos nas lagoas acontece devido à ação da força de gravidade sobre as partículas em suspensão no líquido, desenvolvendo um movimento oblíquo até atingirem o leito da lagoa. O acúmulo destas partículas, segundo Jordão et al. (2014), pode interferir no desempenho do sistema, comprometendo o fluxo projetado e a eficiência na remoção dos poluentes pretendidos.

O acúmulo de sedimentos pode ser caracterizado por meio de um estudo batimétrico, que é uma mensuração topográfica com a finalidade de coletar dados sobre o perfil de elevação em diferentes ambientes. Quando aplicado a lagoas do tratamento de efluentes, proporciona o conhecimento sobre o comportamento do fluido e a deposição de sólidos ao longo do perfil longitudinal das mesmas (ORTIZ et al., 2012).

Com o estudo de elevação da camada de sedimentos em lagoas é possível identificar zonas mortas, de mistura e advectivas. Tal caracterização se deve ao fato de as zonas mortas não apresentarem interação com as zonas advectivas e, portanto, a força da gravidade age por maior tempo sobre os sólidos em suspensão. Tal acontecimento conduz à formação de bancos de lodo com maior espessura quando comparados aos formados em outras regiões das lagoas (ORTIZ et al., 2013).

De forma inversa, as zonas de mistura possuem grande velocidade na troca da massa líquida, assim os sólidos suspensos, quando não há barreiras físicas, são carregados junto com o fluxo do líquido, não havendo tempo hábil para sedimentarem no local. Nestas regiões o volume de lodo depositado geralmente é menor se comparado à zona advectiva (VON SPERLING, 2017). Assim, o objetivo deste estudo é analisar o perfil da camada de sedimentos depositada no leito de uma lagoa facultativa por meio de batimetria, verificando a existência de zonas mortas, de mistura e advectivas. 


\section{MATERIAIS E MÉTODOS}

Com uma população estimada em 94.212 habitantes, o município de Campo Mourão está localizado na mesoregião centro-ocidental paranaense (Figura 1) e tem sua economia baseada na produção agroindustrial, com culturas de ciclo rápido, predominantemente. A densidade populacional é de 123,37 hab $/ \mathrm{km}^{2}$, distribuída em uma área total de 763, 63 km² (IBGE, 2018; IPARDES, 2018). O clima é classificado como CFA: subtropical úmido, tendo verões quentes e baixa frequência de geadas. A temperatura média anual está entre $20^{\circ} \mathrm{C}$ e $21^{\circ} \mathrm{C}$, e os índices pluviométricos se encontram na faixa de 1400 e 1500 milímetros por ano (IPARDES, 2018).

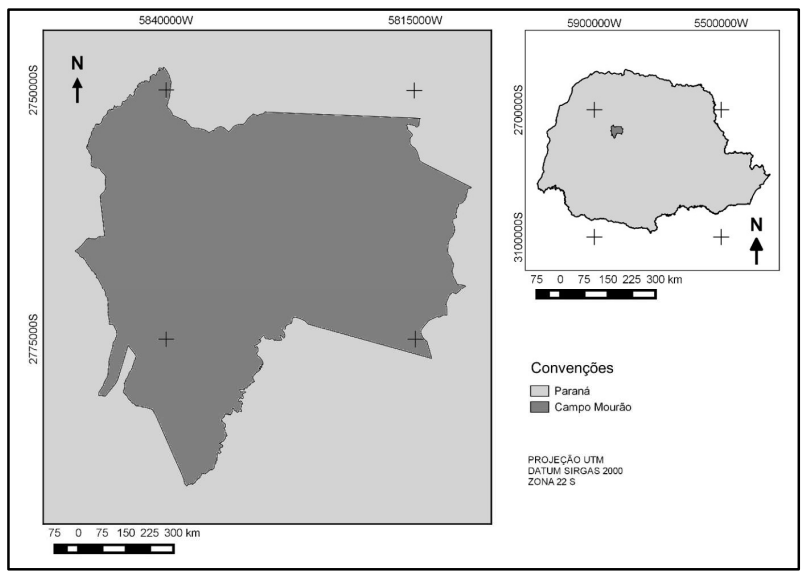

Figura 1: Mapa do município de Campo Mourão e sua posição no estado do Paraná/BR. Fonte: Garcia (2016).

De acordo com o Instituto Brasileiro de Geografia e Estatística (IBGE, 2018), Campo Mourão possui 30.806 domicílios recenseados, dos quais $75 \%$ contam com esgotamento sanitário adequado, ocupando a 43a posição no ranking estadual de saneamento básico. A estação de tratamento de efluentes, objeto desde estudo, é constituída por um sistema composto por tratamento preliminar, primário, secundário e póstratamento. O componente principal responsável pela eliminação dos poluentes é um reator anaeróbio do tipo UASB, seguido de lagoa facultativa. O esquema do sistema de tratamento pode ser conferido na Figura 2.

A geometria da lagoa estudada tem formato irregular, impedindo a confecção de uma malha convencional para a coleta dos dados batimétricos. Desta forma, o método desenvolvido para padronizar os pontos de coleta foi com a utilização de uma corda guia na superfície da lagoa, movendo-a cinco metros a cada linha de coleta, paralelamente à margem de entrada do efluente.

Para a operação, a guia foi fixada às margens paralelas aos dutos de entrada, com o auxílio de ganchos de ferro. Após esta fixação, a partir de cinco metros da linha d'água da lagoa se efetuou a primeira coleta, como pode ser observado na Figura 3. As amostras seguintes foram coletadas a cada cinco metros do ponto anterior. Finalizando a primeira linha de pontos, a guia foi recolocada paralelamente aos dutos de entrada, ainda mantendo a distância de cinco metros da linha anterior. 


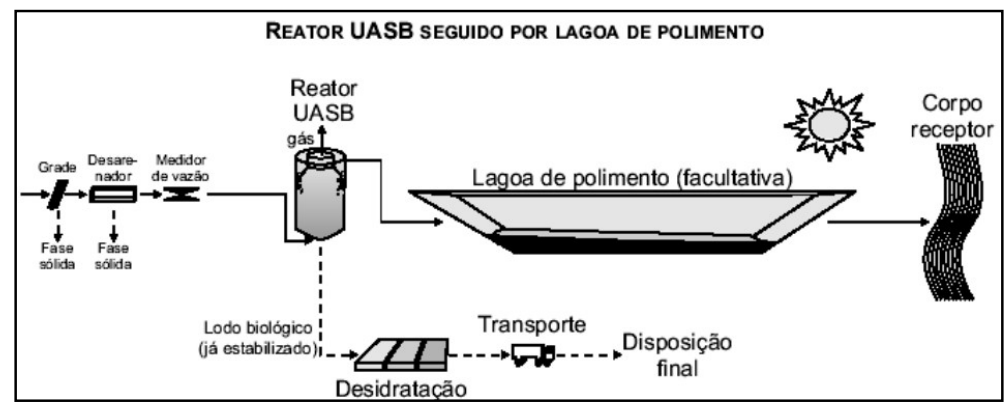

Figura 2: Modelo do sistema de tratamento de efluentes da ETE analisada no município de Campo Mourão/PR. Fonte: Von Sperling (2017).

A lagoa possui uma geometria que se assemelha a uma baía na área de entrada do fluido, em seguida se estende como um retângulo. Na curvatura do perímetro, a movimentação da corda guia aconteceu de forma diferenciada, mantendo-a fixa na margem interna da curva, e percorrendo os cinco metros a cada linha apenas na margem externa (Figura 3). Quando a corda guia tornou-se paralela à margem de saída do fluido, a distância entre linhas passou a ser de dez metros, entretanto, a distância entre pontos se manteve em cinco metros.

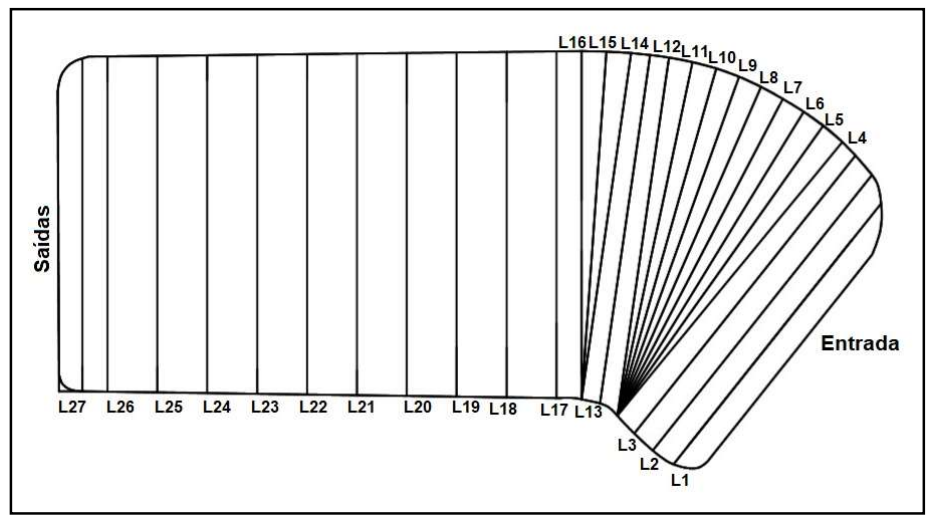

Figura 3: Croqui da disposição das linhas de coleta batimétrica na lagoa facultativa da estação de tratamento de efluentes de Campo Mourão/PR.

Para realizar a coleta dos dados batimétricos foi feito o uso de um bote de alumínio, equipado com remos, empregados com a máxima cautela a fim de evitar a movimentação da massa de lodo na lagoa. A medição foi realizada por meio de uma baliza de aço inoxidável, graduada, com comprimento de três metros, tendo sua extremidade inferior equipada com um disco de aproximadamente 0,15 metros de diâmetro.

Ao encontrar o ponto de coleta, a baliza era introduzida na lagoa, tendo sua velocidade de submersão controlada, até atingir a camada de lodo. Neste instante foi possível observar que o disco na extremidade da baliza sofria resistência para penetrar na massa semissólida. A partir dos dados obtidos na batimetria, desenvolveu-se uma superfície tridimensional que caracterizou a situação encontrada no fundo da lagoa facultativa. Para a operação foi utilizado o software Surfer $8^{\circledR}$, de distribuição gratuita. Para que fosse possível replicar a situação encontrada na lagoa, foi necessário oferecer um tratamento aos dados coletados, atribuindo-Ihes valores para as coordenadas $\mathrm{X}, \mathrm{Y}$ e Z.

A geometria da lagoa foi replicada no software $A u t o C A D^{\circledR}$, onde foi possível desenhar as linhas de coleta e localizar os pontos em cada uma das linhas. Depois de finalizado o desenho do perímetro e linhas de coleta, a geometria foi alinhada ao eixo cartesiano $(0,0)$, permitindo então que se encontrassem as 
coordenadas de cada um dos pontos de coleta. Desta forma, os pontos passaram a ter dimensões X, Y e Z. As informações cartesianas geradas no AutoCAD ${ }^{\circledR}$ foram inseridas nas planilhas do Surfer e possibilitaram então o desenvolvimento da superfície inferior da lagoa. Utilizado as ferramentas deste software foi possível manipular a imagem gerada, graduando-a de acordo com a altura da camada de lodo.

\section{RESULTADOS E DISCUSSÃO}

As linhas de coleta dos dados batimétricos variaram conforme a geometria da lagoa, não sendo possível, portanto, criar uma malha retangular para normatização da coleta. Foram obtidos resultados de 356 pontos em 27 linhas de coleta, conformando uma média de 13 pontos por linha. Para o desenvolvimento dos estudos relacionados ao acúmulo de lodo na lagoa, considerou-se a vazão de operação como a vazão média obtida em projeto $(28,99 \mathrm{~L} / \mathrm{s})$. A maior largura da lagoa foi encontrada no intervalo entre as linhas $\mathrm{L} 3$ e L13, possuindo a dimensão de 70,0 m, enquanto o maior comprimento foi verificado ao final da curvatura externa da lagoa, indo do duto de entrada até a primeira comporta de saída, com a dimensão de 162,0 m.

Com os dados obtidos foi possível verificar que o tempo de detenção do fluido na lagoa (6,27 dias) é aproximadamente 2,75 vezes maior que o tempo de detenção do projeto ( 2,28 dias). Tal fato pode, de acordo com Von Sperling (2017), ser condicionado por problemas operacionais como as zonas de retromistura e as zonas mortas. No primeiro caso, o fluxo, ao encontrar uma barreira, reflete o seu curso e volta a se dirigir à entrada da lagoa, formando uma zona cíclica onde permanece por mais tempo que o calculado em projeto. Já nas zonas mortas, a massa líquida perde velocidade por estar fora da zona de fluxo, se estagnando em áreas periféricas da lagoa. Esta situação contribui com a deposição de sedimentos nestes locais, já que a diminuição da turbulência e velocidade são fatores condicionantes do movimento das partículas de lodo dentro do fluido (MATSUMOTO et al., 2013)

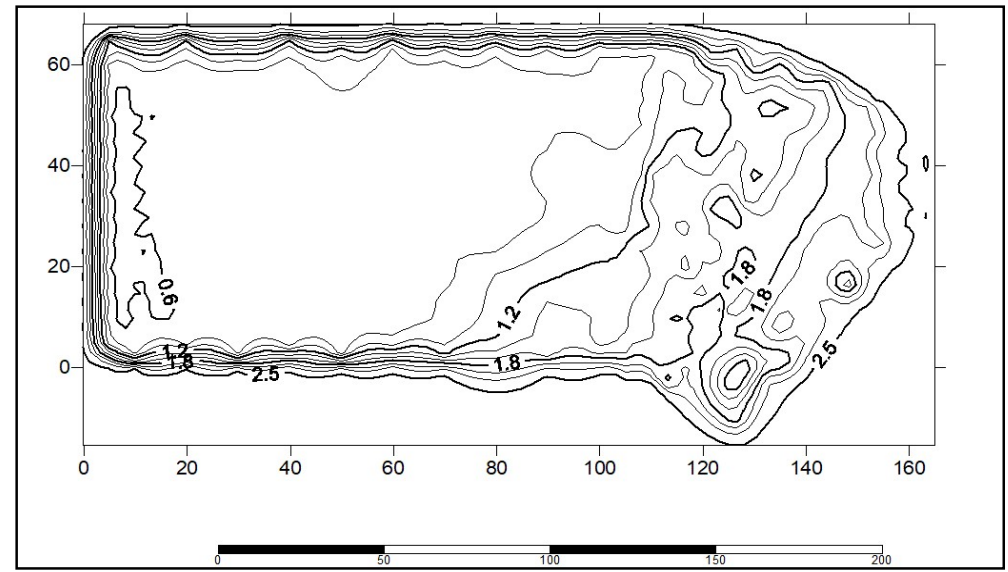

Figura 4: Cotas de altitude da camada de sedimentos depositados no leito da lagoa facultativa localizada na ETE de Campo Mourão/PR.

Pela Figura 4 é possível verificar que o sedimento tem sua maior taxa de sedimentação no início da lagoa, próximo aos dutos de entrada, chegando nestes pontos à altura de 2,5 m, a mesma profundidade da lagoa. O padrão de deposição segue paralelamente à margem de entrada, na forma de um talude, onde a declividade decai gradualmente até atingir a altura que varia entre 0,8 e 0,6 m a partir do meio da lagoa. Há 
de salientar ainda que a sedimentação do lodo acompanha a declividade dos taludes, se mostrando mais intensa próxima às margens (Figura 5), assim como mencionam Ortiz et al. (2013).
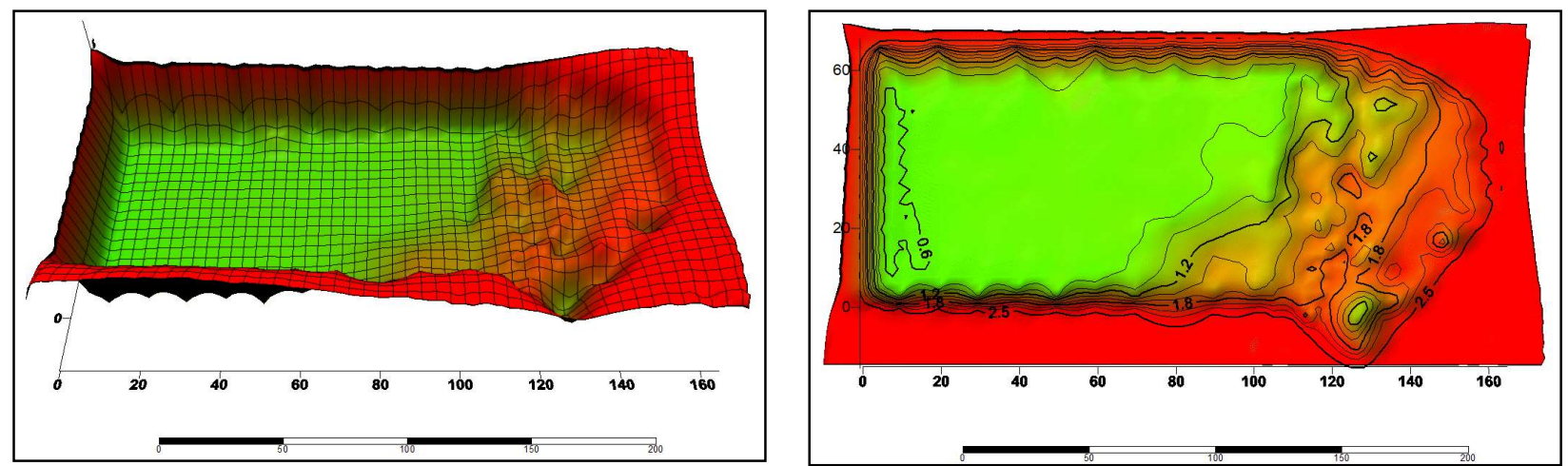

Figura 5: Imagem tridimensional e sobreposição das cotas de altitude da camada de sedimentos depositados no leito da lagoa facultativa da ETE de Campo Mourão/PR.

O modelo de cotas encontrado a partir da batimetria aponta que a maior influência para o acúmulo do lodo no início da lagoa é o seu formato irregular (Figura 5). Tal situação, com base em Souza et al. (2012), se deve ao fato da margem curva se transformar em uma barreira ao fluxo emitido pelos dutos de entrada, assim, quando o líquido adentra a lagoa facultativa, este não tem um escoamento longitudinal livre. Ao se chocar com a margem curva, a velocidade de escoamento é drasticamente reduzida e o fluxo é redirecionado a formar zonas de retromistura. Nesta situação o volume de sólidos se adensa, condicionando a sedimentação e a formação dos bancos de lodo (CASAROTTI et al., 2012).

Da forma como acontece em uma caixa de areia, um tratamento primário comum, quando o fluido consegue seguir pela lagoa, no sentido da saída, a concentração de sólidos em suspensão é consideravelmente inferior, permitindo que o escoamento ocorra sem elevadas taxas de deposição de sedimentos e formação de camadas de lodo muito espessas. Esta situação é observada na segunda metade da lagoa, mais próxima aos dutos de saída (VON SPERLING, 2014).

\section{CONCLUSÕES}

A coleta dos dados batimétricos possibilitou a projeção da camada de sedimentos em uma superfície tridimensional, a qual evidenciou a concentração desigual de sólidos ocasionada por conta da geometria do sistema. Por meio da batimetria conheceu-se de fato a situação operacional da lagoa, sendo possível então atestar a veracidade do modelo tridimensional que representa os sólidos depositados.

Lagoas que recebem efluentes oriundos de tratamentos com lodos ativados têm a característica de acumular sólidos em seu leito a uma taxa elevada, porém este fator é considerado no dimensionamento do sistema, sendo prevista assim a manutenção necessária para a remoção destes sólidos. O que se observou na lagoa analisada é que o formato da construção não condiz com a geometria de projeto, e desta forma o cálculo de deposição de sólidos, no projeto, não é verdadeiro.

O fluxo do efluente sofre grande influência das margens curvas da lagoa, que alteram a linha de corrente, conduzindo o fluido ao movimento circular no leito, efeito este que age sobre as partículas de 
sólidos suspensos e causa sua sedimentação acelerada. Como é possível observar pelas imagens, o local com maior volume de sedimentos se dá justamente no início da lagoa, onde se encontra a região curva.

Conclui-se assim que a deposição de sólidos neste reservatório acontece de maneira irregular, diferente da dimensionada em projeto, e as principais causas para este fenômeno estão relacionadas à geometria da lagoa e a velocidade do fluxo de entrada, que não é elevada o suficiente para conduzir os sólidos em suspensão uniformemente por todo o leito do reservatório.

\section{REFERÊNCIAS}

CASAROTTI, E. G.; MATSUMOTO, T.; ALBERTIN, L. L.. Avaliação da influência do vento na circulação de uma lagoa de estabilização. Engenharia Sanitária e Ambiental, Rio de Janeiro, v.17, n.4, p.401-412, 2012.

GARCIA, L. C.. Análise de viabilidade econômica para implantação de um sistema fotovoltaico on grid na APAE rural de Campo Mourão/PR. Monografia (Graduação em Engenharia Ambiental) - Universidade Tecnológica Federal do Paraná, Campo Mourão, 2016.

IBGE. Instituto Brasileiro de Geografia e Estatística. Cidades: Campo Mourão. Rio de Janeiro: IBGE, 2018.

IPARDES. Instituto Paranaense de Desenvolvimento Econômico e Social. Perfil avançado do município de Campo Mourão. Curitiba: IPARDES, 2018.

JORDÃO, E. P.; PESSOA, C. A.. Tratamento de esgotos domésticos. 4. ed. Rio de Janeiro: Abes, 2014.

LIMA, L. P.; FERREIRA, A. G.; VAZ, L. R. L.; ANDRADE, W. R.; FERREIRA, C. F. S.. Método para seleção de sistemas de tratamento de efluentes em agroindústrias de micro e pequeno porte. Revista Engenharia na Agricultura, Viçosa, v.26, n.4, p.334-342, 2018.
MATSUMOTO, T.; ORTIZ, I. S.. Monitoreo del desempeño y estudio batimétrico de la planta de tratamiento de aguas residuales de Neves Paulista (São Paulo, Brasil). Revista Escuela de Ingenieria de Antioquia, Medelín, v.10, n.20, p.139-151, 2013.

METCALF, L.; EDDY, H. P.. Wastewater Engineering. Treatment, Disposal and Reuse. 3 ed. New York: McGraw Hill, 2015.

ORTIZ, I. A. S.; MATSUMOTO, T.. Evaluación del desempeño de la planta de tratamiento de aguas residuales urbanas de Ilha Solteira (SP) por lagunas facultativas primarias. Ingeniería y Desarrollo, Porto Colombia, v.30, n.2, p.199-222, 2012.

SOUZA, V. A. S.; HELBEL, A. F.; JANZEN, J. G.; DIAS, R. H. S.; COLTRE, H. C. S.. Simulação computacional do comportamento hidrodinâmico em lagoas facultativas com chicanas. Ciência \& Engenharia, Uberlândia, v.21, n.1, p.1521, 2012.

VON SPERLING, M.. Lagoas de estabilização. Belo Horizonte: UFMG, 2017.

VON SPERLING, M.. Introdução à qualidade das águas e ao tratamento de esgotos. 4 ed. Belo Horizonte: UFMG, 2014.

A CBPC - Companhia Brasileira de Produção Científica (CNPJ: 11.221.422/0001-03) detém os direitos materiais desta publicação. Os direitos referem-se à publicação do trabalho em qualquer parte do mundo, incluindo os direitos às renovações, expansões e disseminações da contribuição, bem como outros direitos subsidiários. Todos os trabalhos publicados eletronicamente poderão posteriormente ser publicados em coletâneas impressas sob coordenação da Sustenere Publishing, da Companhia Brasileira de Produção Científica e seus parceiros autorizados. Os (as) autores (as) preservam os direitos autorais, mas não têm permissão para a publicação da contribuição em outro meio, impresso ou digital, em português ou em tradução. 